\title{
BOUNDS FOR LAPLACIAN GRAPH EIGENVALUES
}

\section{A. Dilek MAdEN AND ŞERIFE BÜYÜKKÖSE}

Abstract. Let $G$ be a connected simple graph whose Laplacian eigenvalues are $0=\mu_{n}(G) \leqslant$ $\mu_{n-1}(G) \leqslant \cdots \leqslant \mu_{1}(G)$. In this paper, we establish some upper and lower bounds for the algebraic connectivity and the largest Laplacian eigenvalue of $G$.

Mathematics subject classification (2010): 05C50, 15A18.

Keywords and phrases: algebraic connectivity, Laplacian eigenvalues, trace.

\section{REFERENCES}

[1] W. N. Anderson, T. D. Morley, Eigenvalues of the Laplacian of a graph, Linear Multilinear Algebra 18 (1985), 141-145.

[2] D. TAŞCI Ş. BÜYÜKKÖSE, Bounds for singular values using traces of matrices, Selçuk University Art and Science Faculty, Journal of Science, Konya, Turkey 17 (2000), 137-146.

[3] F. R. K. Chung, Eigenvalues of Graphs, In: Proceeding of the International Congress of Mathematician, Zürich, Switzerland, 1994, 1333-1342.

[4] K. CH. DAS, An improved upper bound for Laplacian graph eigenvalues, Linear Algebra Appl. 368 (2003), 269-278.

[5] M. FiedLeR, Algebraic connectivity of graphs, Czechoslovak Math. J. 23 (1973), 298-305.

[6] J.-S. LI, X.-D. ZHANG, A new upper bound for eigenvalues of the Laplacian matrix of a graph, Linear Algebra Appl. 265 (1997), 93-100.

[7] J.-S. LI, X.-D. ZhANG, On Laplacian eigenvalues of a graph, Linear Algebra Appl. 285 (1998), 305-307.

[8] M. LU, L. ZHANG, F. TIAN, Lower bounds of the Laplacian spectrum of graphs based on diameter, Linear Algebra Appl. 420 (2007), 400-406.

[9] R. Merris, A note on Laplacian graph eigenvalues, Linear Algebra Appl. 285 (1998), 33-35.

[10] R. MERRIS, Laplacian matrices of Graphs: a Survey, Linear Algebra and its Applicaitons 197 (1994), $143-176$.

[11] B. Mohar, The Laplacian spectrum of graphs, In: Graph Theory, Combinatorics and Applications 2 (1998), 871-898.

[12] O. Rojo O., R. Soto, H. Rojo, An always nontrivial upper bound for Laplacian graph eigenvalues, Linear Algebra Appl. 312 (2000), 155-159.

[13] X.-D. Zhang, J.-S. Li, On the $k$-th Largest Eigenvalue of the Laplacian Matrix of a Graph, Acta Math. Appl. Sinica (English Ser.) 17, 2 (2001), 183-190. 